\title{
Vernetzte Medizin zum Anfassen
}

Wie funktioniert eigentlich Telemedizin? Welche Vorteile haben Ärzte, die damit arbeiten? Wie profitieren Patienten? Mit solchen Fragen befasst sich die Sonderschau FutureCare bei der diesjährigen CeBIT in Hannover.

- Anhand verschiedener realistisch nachgestellter Anwendungen wird bei einer CeBIT-Sonderschau (2.-6. März, Halle 9) gezeigt, wie sich Ärzte in Zukunft durch vernetztes Arbeiten noch mehr um den Patienten kümmern können.

\section{Telemedizin auch für die Praxis}

Verschiedene Szenarien werden gezeigt, z.B die Tertiärprävention am Beispiel eines COPD-Patienten. Durch die telemedizinische Betreuung kann er gleichermaßen zu Hause und in der Praxis unterstützt werden.

Weitere Vorführungen demonstrieren, wie Daten Auskunft über den Gesundheitszustand eines Menschen geben und wie diese Daten für weitere Entscheidungen für eine optimale gesundheitliche Versorgung rechtzeitig zur Verfügung stehen. Anhand einer gut nachvollziehbaren und zugleich medizinisch relevanten Geschichte wird der Einsatz von Techniken wie der elektronischen Gesundheitskarte, dem E-Rezept, dem Notfalldatensatz oder der elektronischen Gesundheitsakte verständlich und zugleich fachlich versiert demonstriert.

\section{Kostenlose E-Tickets}

Für die CeBIT steht der „Ärzte Zeitung“ als Medienpartner der begleitenden Messe TeleHealth ein Kontingent kostenloser E-Tickets zur Verfügung. Ein Link auf www.aerztezeitung.de im Kasten "TeleHealth / CeBIT 2010“ in der Navigationsspalte rechts führt zur Registrierung. Das Ticket wird an die angegebene E-Mail-Adresse versandt. Der Ausdruck genügt für den Eintritt.

THOMAS JUNGBLUT .

\section{Die Vorteile in den einzelnen medizinischen Fachgebieten Telemedizin schon praxistauglich?}

\begin{abstract}
MMW: Herr Professor Korb, bringt die Telemedizin Fachärzten zusätzliche Konkurrenz?

Korb: Niedergelassene Ärzte haben sich bisher in diesem Bereich eher konservativ verhalten. Das Thema ist durch die Diskussion um die eGK ja auch eher negativ belegt. Unsere Erfahrungen sind so, dass Ärzte, die in solche Projekte hineinkommen, zunächst Vorbehalte haben und dann später von den Vorteilen überzeugt sind. Der stärkste Effekt einer intensiven Fernbetreuung ist ja meist, dass die Krankenhauseinweisungen zurückgehen. Da haben niedergelassene Ärzte wenig zu fürchten.
\end{abstract}

MMW: Wer muss sich besonders stark auf veränderte Abläufe einstellen? Wie hoch sind die erforderlichen Investitionen?

Korb: Die notwendigen Investitionen liegen für niedergelassene Ärzte der meisten Fachgruppen - wenn sie einmal die auf mittlere Sicht ohnehin erforderliche gute Onlineanbindung haben - bei nahe null. Der klassische Bereich für eine Fernbetreuung von Patienten ist natürlich die Kardiologie, etwa für Patienten mit Herzschrittmachern und Herzrhythmusstörungen sowie bei Herzinsuffizienz. Den Radiologen geht es bei der Telemedizin vor allem darum, Bilder zu verschicken. Eine Fernbefundung von CT-Bildern in speziellen Zentren ist an vielen Orten schon selbstverständlich, teleradiologisch aktive Praxen und Praxisverbünde gibt

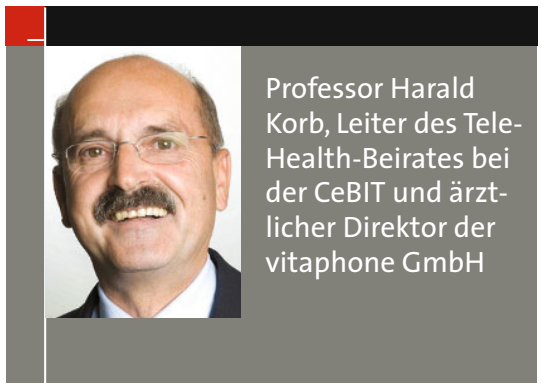

es ebenfalls viele. Auch die Neurologen sind in Sachen Telemedizin ziemlich gut aufgestellt. Das Schlaganfall-Netz Tempis, das in Bayern gestartet worden ist, ist ein exzellentes Programm. Bei den Urologen und Nephrologen gibt es Bestrebungen, die Heimdialyse von Nierenpatienten telemedizinisch zu überwachen.

- Interview: Hauke Gerlof 